React i ve- Medi um I nst abi I i ty of the Space Char ge have of I on Beam i n a Pl asna

\begin{tabular}{|l|l|}
\hline $\begin{array}{l}\text { j our nal or } \\
\text { publ i cat } \mathrm{i} \text { on } \mathrm{t} \text { i t l e }\end{array}$ & 福井大学工学部研究報告 \\
\hline vol une & 26 \\
\hline number & 1 \\
\hline page $r$ ange & $67-71$ \\
\hline year & 1978 03 \\
\hline URL & ht t p: / hdl . handl e. net /10098/4438 \\
\hline
\end{tabular}




\title{
Reactive-Medium Instability of the Space Charge Wave of Ion Beam in a Plasma
}

\author{
Toshitaka IDEHARA, Kuni NAKAJIMA ${ }^{*}$ and Yoshio ISHIDA*
}

(Received Dec. 15, 1977)

The mesh-excited space charge wave of ion beam is observed to be amplified in a plasma, as the result of the reactivemedium instability. The amplification factor has the maximum value at the frequency corresponding to the synchronization condition between the beam wave and the lower hybrid wave mode of plasma, and then, an appreciable deceleration of beam is expected from the observation of the decrease of the phase velocity of amplifying wave.

Recently, the instability of wave in an ion beam-plasma system ${ }^{1)-4)}$ and its nonlinear development ${ }^{5), 6)}$ are investigated with a great interest, because of not only the physical importance of their mechanism but the possibility of its application to the ion heating. In the reports of experimental studies in the system, several authors have shown that the spontaneous excitation of waves is observed in the frequency regions near the ion cyclotron frequency ${ }^{3)}$, 4 ) and its harmonics, 1),2),5) which result from the coupling of the beam wave to the ion cyclotron wave of plasma. Moreover, it is reported that the strong excitation of such wave results in the wave heating of ions. ${ }^{6)}$ In this paper, we report the behavior of the test wave of ion beam excited externally by mesh grid and compare the experimental results with the theoretical consideration.

The experimental apparatus used here is shown in Fig. 1. A vacuum chamber is constructed by using pylex glass tube, whose diameter

*) Department of Applied Physics 
and length are $9.5 \mathrm{~cm}$ and $220 \mathrm{~cm}$, respectively.

The left hand side of the chamber is an ion beam source and the right hand side of it is the region of measurement, both regions being connected through a orifice (denoted by 6 ) whose diameter and length are $8 \mathrm{~mm}$ and $10 \mathrm{~mm}$. The beam source consists of a dc discharge region and an ion beam generated region. By using the cathode (denoted by 2 ) and the anode (denoted by 3 ), the dc discharge is done.
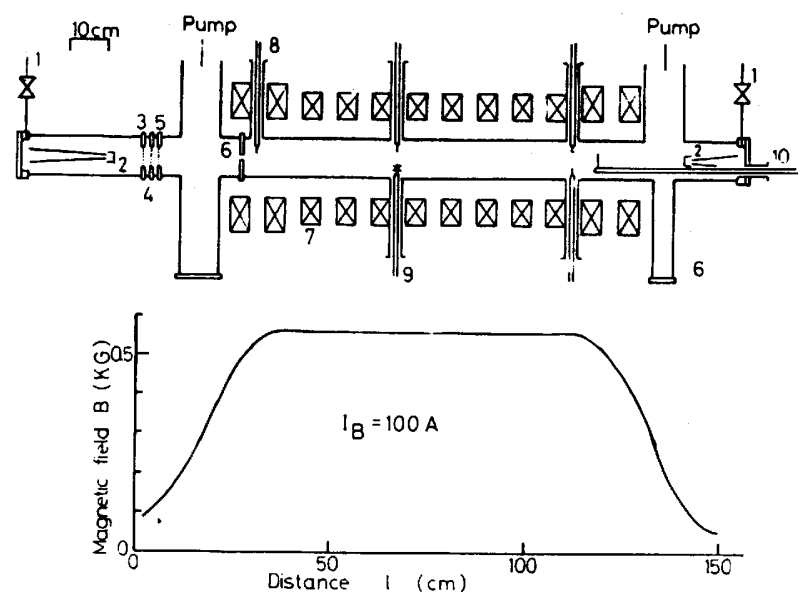

Fig. I The experimental apparatus and the intensity distribution of the magnetic field on the axis. 1. gas inlet, 2 oxide coated cathode, 3. anode, 4. first grid, 5. second grid, 6. orifice, 7. coils, 8. r-probe, 9. asterisk probe, 10. z-probe and 1l. gas inlet.

Ar or He gas is fed

into the chamber through two needle valves (denoted by 1 and 11).

By using the differential pumping, the pressures of three regions, i.e., the dc discharge region, the ion beam generated region and the region of measurement are maintained at $5.0 \times 10^{-4}, 1.0 \times 10^{-5}$ and $1.0 \times 10^{-4}$, respectively. The discharge current $I_{\mathrm{d}}$ is varied from $100 \mathrm{~mA}$ to $3 \mathrm{~A}$. The second grid (denoted by 5 ) in the ion beam generated region is biased negatively to the anode, so that an ion beam is drawn out from the plasma produced by dc discharge through 137 orifices of the anode whose diameter is $3 \mathrm{~mm}$. The acceleration voltage $\mathrm{V}_{\mathrm{a}}$ is varied from $50 \mathrm{~V}$ to $200 \mathrm{~V}$.

An external magnetic field is applied along the tube axis by 12 main coils (denoted by 7) and its intensity distribution on axis is shown in the lower diagram of Fig. 1. The coil current $I_{B}$ is varied up to $300 \mathrm{~A}$. The ion beam travels along the line of force near the axis of the chamber and is injected into the region of measurement through the orifice (denoted by 6 ).

The energy distribution function of ion beam is measured by Farady 


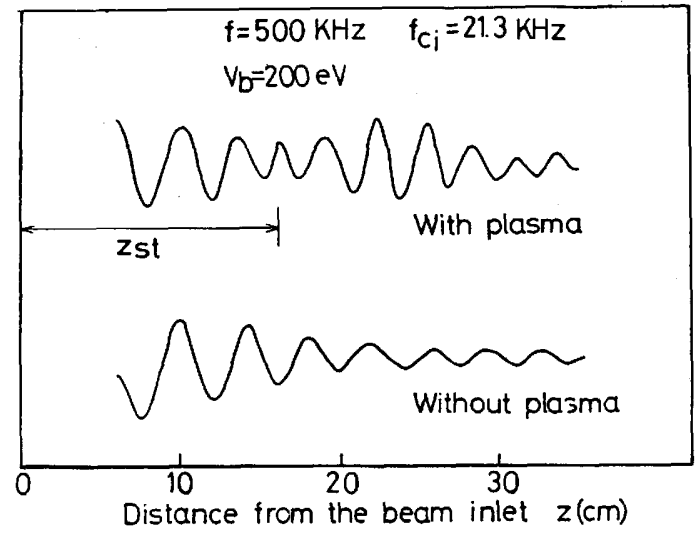

Fig. 2 The propagating wave patterns.

cup. As the energy $E_{b}$ and the density $n_{b}$ of ion beam can be varied independently and its temperature $\mathrm{T}_{\mathrm{b}}$ is relatively low $\left(\mathrm{T}_{\mathrm{b}} \sim 0 . \mathrm{leV}\right)$, the source is suitable one for the investigation of wave phenomenon in an ion beam-plasma system.

The plasma is produced in the region of measurement by dc discharge between the oxide-coated cathode (denoted by $2^{\prime}$ ) and the anode (denoted by 6 ). When the ion beam is injected, the ion beam-plasma system isgenerated in the region.

The space charge wave of ion beam is excited by applying the signal to the first grid, whose frequency $f$ is varied from $400 \mathrm{KHz}$ to $700 \mathrm{KHz}$. The propagating wave pattern along the $z$ axis in the region of measurement is observed by the interferometer system, the result of which is shown in Fig. 2. The lower pattern corresponds to the case where the dc discharge is not done, so that only an ion beam travels in a vacuum. It is seen that the space charge wave whose phase velocity is nearly equal to that of ion beam $\left(\omega / k=v_{b}\right)$ propagates with small damping. The upper pattern shows that in the ion beam-plasma system when the dc discharge is done. The amplifying wave appears in the region sufficiently far from the beam inlet $\left(z>z_{s t}\right)$, while the damping wave is seen near the beam inlet $\left(z<z_{s t}\right)$. 
It is known from the comparison of the amplifying wave with the damping one, that the wave length of the former is shorter than that of the latter, that is, the wave number component parallel to the field $k_{11}$ of the former is larger. As the frequency $\omega / 2 \pi$ and the wave number $k_{\|}$of the space charge wave must satisfies the condition of $\omega / k_{\|}=v_{b}$, the above-mentioned fact may imply that the ion beam slows down in the region of $z>z_{\text {st }}$, as the result of energy transfer from the beam to the amplifying wave.

The wave number components ( $k_{\| r}$ and $k_{\| r}$ ) of the damping wave and amplifying wave, the growth rate $k_{11 i}$ of the latter wave and the starting length $z_{\text {st }}$ can be estimated from both wave patterns in Fig. 2. The results are shown in Fig. 3, as functions of the frequency $f$. At the frequency ( $f \sim 600 \mathrm{KHz}$ ) where the maximum growth rate is observed, the phase velocity of the amplifying wave $\omega / k_{\| r}$ and starting length $z_{\text {st }}$ become minimum which implies that the energy transfer from the beam to the wave occurs most effectively at the frequency. On the other hand, the phase velocity of the damping wave $\omega / k_{\|_{r}}^{\prime}$ is independent on the frequency and equal to the initial beam velocity estimated from the acceleration voltage $V_{a}$. In this case, as the matter of course, the energy trnsfer does not occur.

The results are explained by the reactive-medium instability of the space charge wave of ion beam near the lower hybrid frequency, which is described by the following dispersion relation,

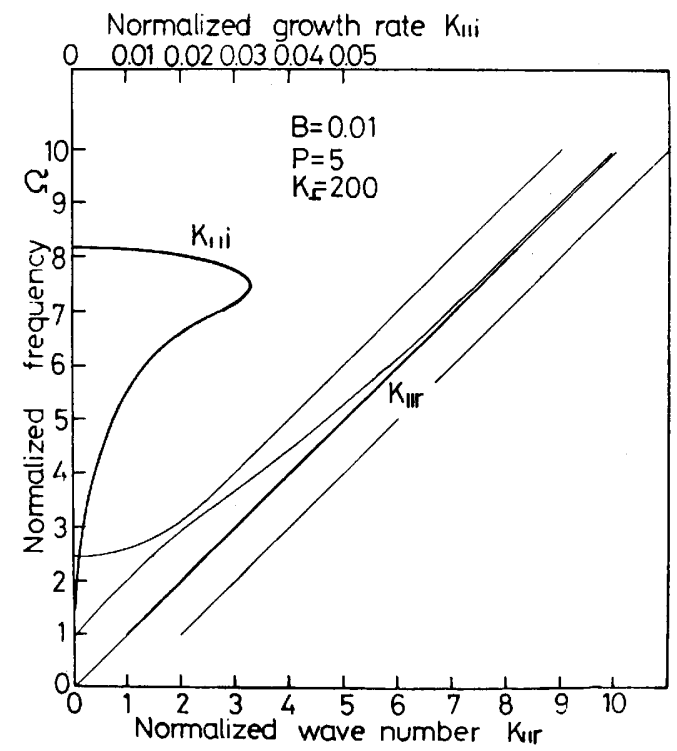

Fig. 4 The calculated dispersion relation. It is seen that the space charge wave of beam is unstable in the frequency region

$$
\begin{array}{ll}
k_{\perp}^{2}\left(1-\frac{\omega_{p i}^{2}}{\omega^{2}-\omega_{c i}^{2}-\frac{\omega_{p e}^{2}}{\omega^{2}-\omega_{c e}^{2}}} \quad \begin{array}{l}
\text { lower than the lowe } \\
\text { mode of plasma. }
\end{array}\right. \\
\left.-\frac{\omega_{b i}^{2}}{\left(\omega-k_{11} v_{b}\right)^{2}-\omega_{c i}^{2}}\right)+k_{\| 1}^{2}\left(1-\frac{\omega_{p e}^{2}}{\omega^{2}}-\frac{\omega_{b i}^{2}}{\left(\omega-k_{\|} v_{b}\right)^{2}}\right)=0 .
\end{array}
$$


For the typical values of the normalized quantities $B=\omega_{b i}^{2} / \omega_{p i}^{2}=0.01$, $\mathrm{P}=\omega_{\mathrm{pi}}^{2} / \omega_{\mathrm{ci}}^{2}=5$ and $\mathrm{K}_{\perp}=\mathrm{k}_{\perp} \mathrm{v}_{\mathrm{b}} / \omega_{\mathrm{ci}}=200$, the above relation is calculated numerically, by assuming the real frequency $\Omega=\omega / \omega_{c i}$ and the complex wave number $\mathrm{k}_{11}=\mathrm{k}_{11} \mathrm{v}_{\mathrm{b}} / \mathrm{w}_{\mathrm{ci}}$. The result is shown in Fig. 4, which agrees with the experimental results shown in Fig. 3, qualitatively.

In conclusion, the reactive-medium instability of the space charge wave of ion beam is observed near the lower hybrid frequency and the energy transfer from beam to the wave may be verified from the measurement of slowing down of the wave. This work is supported by the Grant-inAid from the Ministry of Education of Japan.

\section{References}

1) S. Seiler, M. Yamada and H. Ikezi, Phys. Rev. Letters 37 (1976) 700 .

2) H. Böhmer, Phys. of Fluids 19 (1976) 1371.

3) P. Michelsen, H. L. Pécseli, J. Juul Rasmussen and N. Sato, Phys. of Fluids 19 (1976) 453.

4) P. Michelsen, H. L. Pécseli and J. Juul Rasmussen, Phys. of Fluids 20 (1977) 866.

5) H. Böhmer, J. P. Hauck and N. Rynn, Phys. of Fluids 19 (1976) 450.

6) D. L. Correll, H. Böhmer, N. Rynn and R. A. Stern, Phys. of Fluids 20 (1977) 822 . 
